\title{
Compact Charged Stars
}

\author{
Beatriz B. Siffert , J.R.T. de Mello Neto, and Maurício O. Calvão \\ Instituto de Física, Universidade Federal do Rio de Janeiro, \\ Caixa Postal 68528, 21941-972, Rio de Janeiro, RJ, Brazil
}

\section{Received on 24 October, 2006}

\begin{abstract}
We investigate the possibility that charged compact objects could be the accelerators of high energy cosmic rays. In order to do so, we choose to first solve numerically a system of differential equations describing the structure of charged compact objects, including the generalization of the Tolman-Oppenheimer-Volkoff equation for this class of objects. We assume a polytropic equation of state for the fluid and, for simplicity, a linear relation between charge density and the fluid energy density. We obtain upper limits for the charge such objects can acquire and study the stability of these equilibrium configurations.
\end{abstract}

Keywords: Compact objects; Tolman-Oppenheimer-Volkoff equations; Cosmic rays acceleration

\section{INTRODUCTION}

The possibility that stars could actually contain a nonvanishing net charge was first pointed out by Rosseland [1] in 1924. He modeled the star as a gas of positive ions and electrons and concluded that, due to their greater kinetic energy, the electrons tend to escape from the star more often than the ions. The star will then acquire a net positive charge. The process will be carried on until the electric field induced in the star stops more electrons from escaping. Harrison and Bally [2] further showed that the maximum electric charge a star can acquire through this process should be $\sim 100\left(M / M_{\odot}\right)$ C.

Recently other mechanisms to induce electric charge into compact objects, in particular into black holes, have been proposed. In this paper it is not our concern to study these mechanisms. We intend only to study the effects charge would cause on these objects, and their potential applicability in acceleration of cosmic rays to very high energies.

The highest energy cosmic rays we detect on Earth reach us with $\sim 10^{20} \mathrm{eV}$. A naïve energy conservation estimation of the charge $Q$ a compact object must enclose in order to produce an electric field capable of accelerating a proton to this energy provides:

$$
Q \sim 10^{19}\left(r / R_{\odot}\right) \mathrm{C}
$$

where we assume the ideal case of no losses during propagation and $r$ is the distance of closest aproximation between the object an the proton.

To get a feeling about this value, which, for ordinary situations, is very high compared to the $\sim 100\left(M / M_{\odot}\right) \mathrm{C}$, we analyze a typical constraint on the charge a black hole can have, by demanding that the singularity is not naked. In the Reissner-Nordstrøm spacetime, this requirement sets $Q^{2} \leq$ $M^{2}$ and the maximum charge a black hole can have is:

$$
Q_{\max } \sim 10^{20}\left(M / M_{\odot}\right) \mathrm{C} .
$$

If a black hole could really acquire such a huge charge and stay stable, it would be a very strong candidate for an high energy cosmic ray accelerator.
In this work we study what effects a non-vanishing net charge could cause in the structure of compact stars in general. We also analyze the stability of such charged objects, trying to determine if they could really exist in a stable configuration in nature.

\section{THE STRUCTURE OF CHARGED COMPACT STARS}

The generalization of the Tolman-Oppenheimer-Volkoff equation for a charged star was proposed in 1971 by Bekenstein [3], who also pointed out many arguments against the stability of such stars. Most of the formalism shown in this section is on the format presented by Ray et al. [4], whose results we wanted to reproduce at first.

We take the line element for a spherically symmetric and static star:

$$
d s^{2}=e^{v} c^{2} d t^{2}-e^{\lambda} d r^{2}-r^{2}\left(d \theta^{2}+\sin ^{2} \theta d \phi^{2}\right)
$$

and model the matter inside the star as a perfect fluid plus an electromagnetic field:

$$
T_{v}^{\mu}=(P+\varepsilon) u^{\mu} u_{v}-P \delta_{v}^{\mu}+\frac{1}{4 \pi}\left(F^{\mu \alpha} F_{\alpha v}-\frac{1}{4} \delta_{v}^{\mu} F_{\alpha \beta} F^{\alpha \beta}\right),
$$

where $P$ is the pressure, $\varepsilon$ is the energy density of the fluid and the electromagnetic tensor is given by:

$$
F_{\alpha \beta}=\frac{\partial A_{\beta}}{\partial x^{\alpha}}-\frac{\partial A_{\alpha}}{\partial x^{\beta}},
$$

satisfying Maxwell's equations:

$$
\frac{1}{\sqrt{-g}} \frac{\partial}{\partial x^{\beta}}\left(\sqrt{-g} F^{\alpha \beta}\right)=4 \pi j^{\alpha} .
$$

Next we solve Einstein's equations, using the following quantities:

- A spherical surface of radius $r$, within the star, presents an electric field given by:

$$
E(r)=\frac{1}{\varepsilon_{0} r^{2}} \int_{0}^{r} r^{\prime 2} \rho_{c h} e^{\lambda / 2} d r^{\prime}
$$


- It encloses an electric charge:

$$
Q(r)=\int_{0}^{r} r^{\prime 2} \rho_{c h} e^{\lambda / 2} d r^{\prime},
$$

where $\rho_{c h}$ is the star's charge density.

- The star's mass inside a spherical shell of radius $r$ is therefore:

$$
M(r)=\frac{4 \pi}{c^{2}} \int_{0}^{r} r^{\prime 2}\left(\varepsilon+\frac{\varepsilon_{0} E^{2}}{2}\right) d r^{\prime} .
$$

The (00) component of Einstein's equations gives:

$$
\frac{e^{-\lambda}}{r^{2}}\left(r \frac{d \lambda}{r}-1\right)+\frac{1}{r^{2}}=\frac{8 \pi G}{c^{4}}\left(\varepsilon+\frac{\varepsilon_{0} E^{2}}{2}\right)
$$

which can be rewritten in the following form:

$$
\frac{d}{d r}\left[r\left(1-e^{-\lambda}\right)\right]=\frac{8 \pi G}{c^{4}} r^{2}\left(\varepsilon+\frac{\varepsilon_{0} E^{2}}{2}\right) .
$$

Integrating with respect to $r$, we obtain:

$$
e^{-\lambda}=-\frac{2 G}{c^{2} r} M(r)+1 \text {. }
$$

Using these expressions, the four differential equations for the equilibrium of charged stars turn out to be the aforementioned generalized Tolman-Oppenheimer-Volkoff equation:

$$
\frac{d P}{d r}=-\frac{G\left[M+4 \pi r^{3}\left(\frac{P}{c^{2}}-\frac{\varepsilon_{0} E^{2}}{2 c^{2}}\right)\right](\varepsilon+P)}{c^{2} r^{2}\left(1-\frac{2 G M}{c^{2} r}\right)}+\rho_{c h} E e^{\lambda / 2}
$$

and

$$
\begin{gathered}
\frac{d E}{d r}=-\frac{2 E}{r}+\frac{\rho_{c h} e^{\lambda / 2}}{\varepsilon_{0}} \\
\frac{d M}{d r}=4 \pi r^{2}\left(\frac{\varepsilon}{c^{2}}+\frac{\varepsilon_{0} E^{2}}{2 c^{2}}\right), \\
\frac{d \lambda}{d r}=\frac{8 \pi G}{c^{2}} r e^{\lambda}\left(\frac{\varepsilon}{c^{2}}+\frac{\varepsilon_{0} E^{2}}{2 c^{2}}\right)-\left(\frac{e^{\lambda}-1}{r}\right) .
\end{gathered}
$$

Since we have 6 variables, $P, E, M, \lambda, \rho_{c h}$ and $\varepsilon$, and 4 equations, we need two other equations in order to solve the system of eqs. (13)-(16). One of them relates the charge density $\rho_{c h}$ and the fluid energy density $\varepsilon$. It is actually not easy to guess what this relation should be, so we use, for simplicity, the relation suggested by [4]:

$$
\rho_{c h}=f \varepsilon, \quad f=\text { const. }
$$

A polytropic equation of state will play the role of the other missing equation:

$$
P=\kappa\left(\frac{\varepsilon}{c^{2}}\right)^{\Gamma}
$$

We model the interior of the star as a non-relativistic Fermi gas of nuclei and electrons [5]. In this case the exponent $\Gamma$ is $5 / 3$ and $\kappa$ is given by:

$$
\kappa=\frac{\hbar^{2}}{15 \pi m_{e}}\left(\frac{3 \pi^{2} Z}{A m_{N}}\right)^{5 / 3} .
$$

The value of the constant $\kappa$ depends on the type of ions that form the star. We again follow the steps of [4] and choose $\kappa=0.05 \mathrm{fm}^{8 / 3}(c=\hbar=1)$, which corresponds in SI units to $\kappa=8.7 \times 10^{3} \mathrm{~kg}^{-2 / 3} \mathrm{~m}^{4} / \mathrm{s}^{2}$.

\section{SOLVING THE EQUATIONS}

Inserting eqs. (17) and (18) into eqs. (13)-(16) we end up with a system of four coupled differential equations with four variables, namely $P(r), E(r), M(r)$ and $\lambda(r)$, which can be solved numerically. The boundary conditions are given by:

$$
E(r=0)=M(r=0)=\lambda(r=0)=0 \text { and } P(r=0) \equiv P_{c} \text {. }
$$

Here the boundary condition for $\lambda$ arises from eqs. (12) and (15), via L'Hôpital's rule.

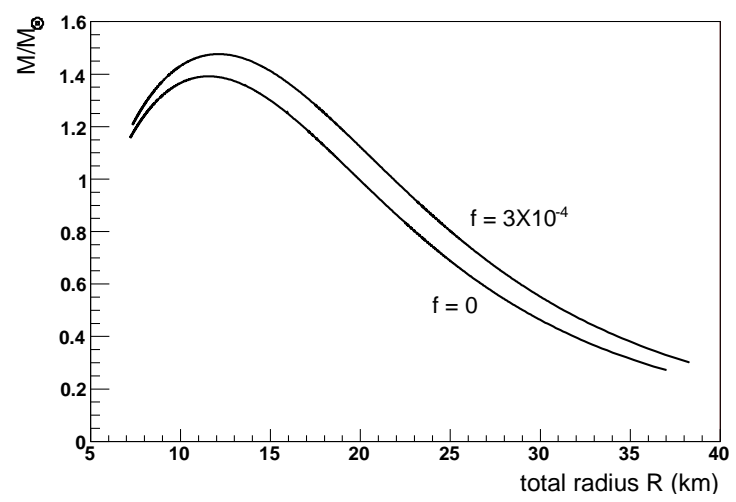

FIG. 1: Total mass $\times$ total radius for different values of $f$ (in units of $\left.\left(\mathrm{MeV} / \mathrm{fm}^{3}\right)^{1 / 2} / \mathrm{km}\right)$. The stars on the right side of the graphic have the smallest values of $P_{c}$, while the ones on the left side have the largest ones.

The parameters we have control over are $P_{c}$ and $f$, the proportionality constant in eq. (17), since we fix $\Gamma=5 / 3$ and $\kappa=0.05 \mathrm{fm}^{8 / 3}$. For each pair $\left(P_{c}, f\right)$ there is a different solution.

The typical densities inside neutron stars are of the order of the nuclear density:

$$
\rho_{\text {nис }} \simeq 10^{14} \mathrm{~g} / \mathrm{cm}^{3} .
$$

More compact objects such as black holes have densities of a few orders of magnitude higher. We integrate the equations for values of $P_{c}$ within the range $5 \times 10^{-2} \mathrm{MeV} / \mathrm{fm}^{3} \leq P_{c} \leq$ $5 \times 10^{3} \mathrm{MeV} / \mathrm{fm}^{3}$, which corresponds to $1.5 \times 10^{13} \mathrm{~g} / \mathrm{cm}^{3} \leq$ $\rho_{c} \leq 1.5 \times 10^{16} \mathrm{~g} / \mathrm{cm}^{3}$. 
Figure 1 shows the total mass against the radius obtained for stars with the $P_{c}$ values specified and two different values of $f$. The case $f=0$ corresponds to an uncharged star. We see that the effects in the structure of the stars due to the presence of charge begin to be important for $f \sim 3 \times 10^{-4}\left(\mathrm{MeV} / \mathrm{fm}^{3}\right)^{1 / 2} / \mathrm{km}$. While testing various values of $f$, we found out that for values larger than $f=1.14 \times$ $10^{-3}\left(\mathrm{MeV} / \mathrm{fm}^{3}\right)^{1 / 2} / \mathrm{km}$ all the dependent variables numerically diverge, making the system impossible to solve.

\section{RESULTS}

Figure 2 shows the total charge enclosed by the stars against their radius, obtained for the same range of $P_{c}$ used in Figure 1 . We see that these configurations yield charges of $\sim 10^{20}$ $\mathrm{C}$, much larger than the $\sim 100\left(M / M_{\odot}\right) \mathrm{C}$ estimated to exist due to the process described in section I.

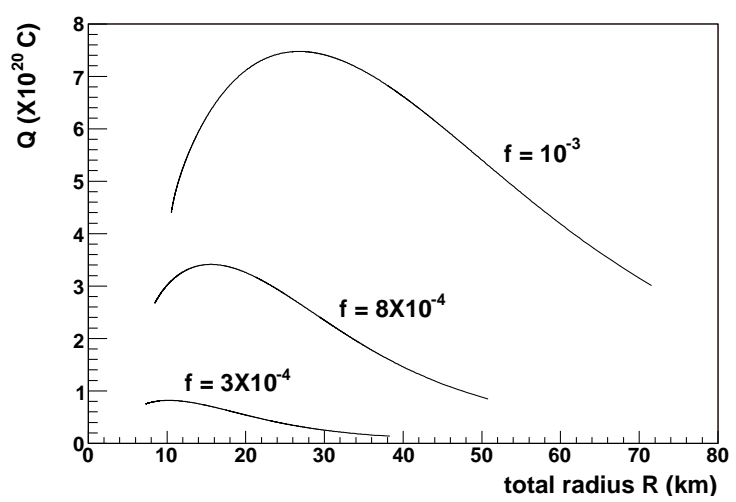

FIG. 2: Total charge $\times$ total radius.

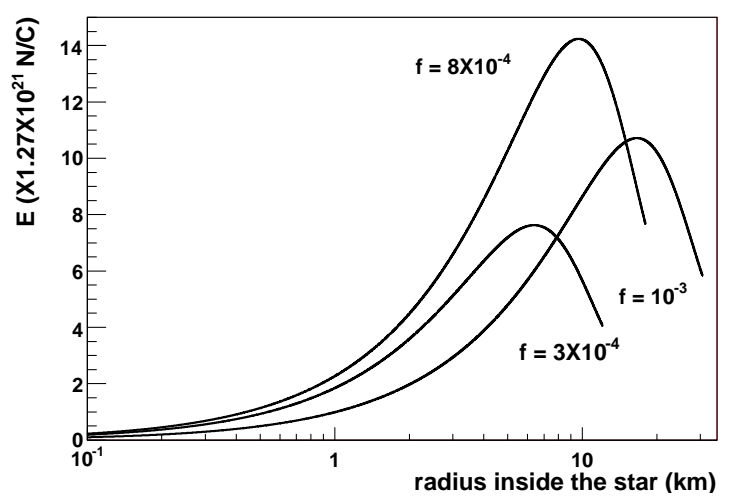

FIG. 3: Total electric field on the surface $\times$ radius inside the star.

Figure 1 shows that there is, for each value of $f$, a maximum mass star. The bevahior of the electric field inside this maximum star is shown in Figure 3. Although the maximum value of the electric field is achieved inside the star, its value on the surface is still very large $\left(\sim 10^{21} \mathrm{~N} / \mathrm{C}\right)$.

The solutions of eqs. (13)-(16) are configurations in hydrostatic equilibrium but are not guaranteed to be stable, which leads us to the next considerations.

\section{A. Stability}

A necessary condition for stability [5] is that, as the central density increases, the configuration satisfying eqs. (13)-(16) must be more massive, i.e.:

$$
\frac{d M\left(\rho_{c}, f\right)}{d \rho_{c}}>0 .
$$

We found out that the more charged a star is, the smaller is the number of stable configurations that can be achieved.

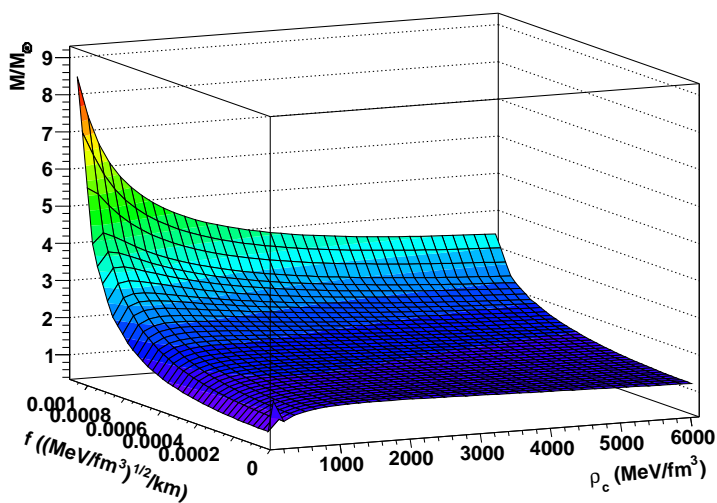

FIG. 4: $M \times \rho_{c} \times f$. Configurations that appear in the portion of the surface contained in the $\rho_{c} \times M$ plane that has positive slope are stable.

This behavior goes on until $f$ reaches its critical value, above which there are no stable configurations at all. These results are shown in Figure 4.

We see that the presence of charge can be associated with instability, in the sense that it is less probable to achieve stability in charged stars than in neutral ones.

\section{B. Charge regeneration}

An interesting effect induced by the presence of charge is the so called "charge regeneration" [3]. At the same time that charge contributes to the expansion of the star (as can be seen in Figures 1 and 2), it also contributes to its collapse, since it increases the total mass (eq. (9)).

The contribution to the gravitational force will overwhelm the contribution to expansion if:

$$
\int_{0}^{r} E^{2} r^{\prime 2} d r^{\prime}>E^{2} r^{3}
$$




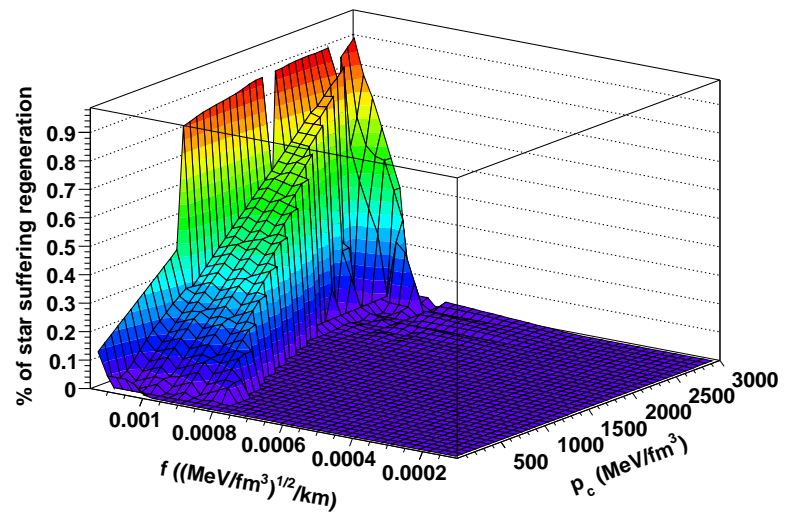

FIG. 5: Fraction of the star suffering regeneration $\times f \times P_{c}$.

We tested this condition in each step of the integration for each pair $\left(P_{c}, f\right)$ studied. Results are shown in Figure 5. We found out that regeneration can occur in some depths of the star and not in others. The $z$ axis of figure 5 represents this feature, showing the percentage of the star that suffers regeneration.

Our attention was called to charge regeneration because we thought it might be related to the stability issue and to the critical value of $f$. But, comparing Figure 4 and 5, we see that there is no correlation between the area where charge regeneration occurs and the instability area. We also see from Figure 5 that charge regeneration occurs for the highest values of $f$, but depends on the value of $P_{c}$, while the critical value for $f$ is the same for all values of $P_{c}$.

\section{The critical value for $f$}

The existence of a critical value for $f$ is reasonable, since it is natural to expect a limit for the amount of charge matter can hold. We do not know however if the value we obtained numerically for this limit is physical. We can only say that the equations cannot be solved for values of $f$ greater than the critical one. To get a feeling about the meaning of this value, let us evaluate the hypothetical case of a star with total mass $M$ and total charge $Q$ in equilibrium, with charge balancing the gravitacional force. For an infinitesimal volume of the star's surface containing charge $q$ and mass $m$ :

$$
k Q q=G M m \Rightarrow k \rho_{c h}^{2}=G \rho^{2} .
$$

For real stars pressure must be taken into account, so eq. (24) should be rewritten as:

$$
k \rho_{c h}^{2}<G \rho^{2},
$$

or

$$
f<\left(\frac{G}{k c^{4}}\right)^{1 / 2}=1.15 \times 10^{-3} \frac{1}{\mathrm{~km}}\left(\frac{\mathrm{MeV}}{\mathrm{fm}^{3}}\right)^{1 / 2} .
$$

This value is suggestively close to the critical value we obtained for $f$. We can therefore interpret it as representing the proportion between charge and energy necessary to support a star in the limiting case where there is no pressure. Since this case is completely unrealistic, we should expect stars to have much lower values for $f$.

\section{CONCLUSION}

We saw that, at least in principle, compact objects can hold huge amounts of charge in stable configurations. But, as a consequence, they have also huge electric fields on their surface. These electric fields are three orders of magnitude larger than the critical eletric field for pair production in vacuum:

$$
E_{\text {critical }} \sim 10^{18} \mathrm{~N} / \mathrm{C},
$$

causing discharge of the objects.

Besides this one, other astrophysical mechanisms, such as the interaction with a nearby plasma, might contribute as well to the loss of charge. This seems to limit the usefulness of these compact objects as realistic accelerators of high energy cosmic rays.

We thank Conselho Nacional de Desenvolvimento Científico e Tecnológico, CNPq.
[1] S. Rosseland, Mont. Not. Royal Astronomical Society 84, 720 (1924).

[2] J. Bally, E. R. Harrison, The Astrophysical Journal, 220, 743 (1978).

[3] J. D. Bekenstein, Phys. Rev. D 4, 2185 (1971).

[4] S. Ray, A. L. Espíndola, M. Malheiro, J. P. S. Lemos, and V. T.
Zanchin, Phys. Rev. D 68, 084004 (2003).

[5] N. K. Glendenning, Compact Stars: Nuclear Physics, Particle Physics and General Relativity, 2nd ed. Springer Verlag, New York (2000). 